\title{
Comunicación
}

\section{Rendimiento productivo de pollos de engorde suplementados con tilosina fosfato o enramicina como promotores de crecimiento}

\author{
Productive performance of broilers supplemented with tylosin phosphate or \\ enramycin as growth promoters
}

\author{
Sandra Espinoza C. ${ }^{1}$, Eliana Icochea D. ${ }^{1,3}$, Pablo Reyna S. ${ }^{1}$, Viviana San Martín ${ }^{1}$, \\ Nelly Giovanna Cribillero B. ${ }^{1}$, Daniel Molina M. ${ }^{2}$
}

\section{Resumen}

Se evaluó el rendimiento productivo de pollos de engorde suplementados con tilosina fosfato y enramicina como promotores de crecimiento hasta los 42 días de edad. Se usaron 400 pollos machos de engorde, distribuidos en 4 tratamientos de 100 aves con 5 repeticiones cada uno. Los tratamientos fueron: T1, control; dieta sin antibiótico; T2, dieta con enramicina $8 \%$ en dosis de $10 \mathrm{ppm}$; T3, dieta con enramicina $8 \%$ en dosis de 5 ppm; T4, dieta con tylosina fosfato $25 \%$ en dosis de $55 \mathrm{ppm}$. Los grupos tratados presentaron un mayor peso corporal y mejor índice de conversión alimenticia e índice de eficiencia productiva europea que el grupo control; sin embargo, no hubo diferencias en ganancia de peso, consumo de alimento y mortalidad.

Palabras clave: tilosina fosfato; enramicina; promotores de crecimiento; pollos de engorde; rendimiento productivo

\section{AbSTRACT}

The productive performance of broilers supplemented with tylosin phosphate and enramycin as growth promoters up to 42 days of age was evaluated. Four hundred male broilers were distributed into 4 treatments of 100 birds with 5 repetitions each. The treatments were: $\mathrm{T} 1$, control; diet without antibiotic; $\mathrm{T} 2$, diet with $8 \%$ enramycin in a

\footnotetext{
${ }^{1}$ Laboratorio de Patología Aviar, Facultad de Medicina Veterinaria, Universidad Nacional Mayor de San Marcos, Lima, Perú

${ }^{2}$ ILENDER Perú S.A., Lima

${ }^{3}$ E-mail: eliana.icochea@gmail.com
}

Recibido: 12 de enero de 2018

Aceptado para publicación: 28 de septiembre de 2018 
dose of $10 \mathrm{ppm}$; T3, diet with enramycin $8 \%$ in a dose of $5 \mathrm{ppm}$; T4, diet with tylosin phosphate $25 \%$ in a dose of $55 \mathrm{ppm}$. The treated groups presented a higher body weight and better feed conversion index and European productive efficiency index than the control group; however, there were no differences in body weight gain, feed intake and mortality.

Key words: tylosin phosphate; enramycin; growth promoters; broilers; productive performance

\section{INTRODUCCIÓN}

Los promotores de crecimiento provocan modificaciones de los procesos digestivos y metabólicos de las aves, lo cual se traduce en mejoras de la eficacia de utilización de los alimentos y de la ganancia de peso y rendimiento productivo de los animales (Sumano y Gutiérrez, 2010; Kim et al., 2012).

En la avicultura, los antibióticos forman parte de la composición de la ración animal, para lo cual pueden actuar con dos fines claramente diferenciados: como terapéuticos o curativos y como profilácticos o promotores para la mejora en el crecimiento y la eficiencia productiva del animal (Dibner y Richards, 2005), ya que ayudan al animal a expresar su potencial genético de crecimiento (Ferket, 2007; Shiva, 2007).

Según el informe de 2007 de la Organización Mundial de la Salud (OMS), la resistencia a los antibióticos es uno de los problemas de salud pública más graves. El aumento de resistencia a los antimicrobianos de bacterias causantes de graves enfermedades en animales de granja presenta una seria amenaza para la producción pecuaria y la salud pública (Acar y Rostel, 2003; Lin, 2014).

La Comisión Europea prohibió en 1997 el uso de avoparcina para no correr riesgos de disminuir la eficacia de un antibiótico de uso humano basado en glicopéptidos (Cepero, 2006). Ese mismo año se estableció un programa de vigilancia sobre la aparición de bac- terias resistentes a antibióticos usados como aditivos alimentarios en cerdos y pollos de engorde en mataderos de seis estados miembros de la Unión Europea (Casewell et al., 2003), y a partir de enero de 2006 se prohibió el uso de promotores de crecimiento en raciones alimenticias para animales (Comisión Europea, 2005).

Los antibióticos de mayor uso para los tratamientos preventivos y curativos en campo son latilosina, latiamulinay la asociación lincomicina-espectinomicina(Cerdá, 2011). La tilosina es un antibiótico pertenecienteal grupo de los macrólidos cuyo espectro de acción se limita activamente contra microorgani smos Gram positivos, siendo usado exclusivamente en medicina veterinaria (Lewicki, 2006, Sumano y Gutiérrez, 2010).

La enramicina es un antibiótico polipeptídico, formado por dos moléculas: la enramicina A y la B, producido por Streptomyces fungicidius. Debido a que la enramicina no es absorbida en el tracto gastrointestinal no existe el riesgo de la presentación de residuos en tejidos (Sumano y Gutiérrez, 2010, FAMIC, 2014). El APC enramicina actúa inhibiendo a las enzimas que utilizan las bacterias del género Clostridium para penetrar en la pared del intestino, siendo este mecanismo único entre los promotores de crecimiento (Vargas, 2010).

El presente estudio evaluó la efectividad de la adición de enramicina y tilosina fosfato en la ración de pollos de engorde sobre el comportamiento productivo de las aves. 


\section{Materiales y Métodos}

El estudio se llevó a cabo en el galpón experimental del Laboratorio de Patología Aviar de la Facultad de Medicina Veterinaria, Universidad Nacional Mayor de San Marcos, Lima. La fase experimental se desarrolló entre enero y marzo de 2013.

Se calculó el tamaño muestral para una diferencia de medias en muestras independientes mediante al paquete estadístico $\mathrm{R} v$. 3.3.1 con un poder de la prueba de $80 \%$ y un nivel de significancia de $5 \%$, resultando en al menos cuatro unidades experimentales (repeticiones) por tratamiento, de allí que se utilizaron 400 pollos de engorde, machos, de un día de edad, de la línea Cobb Vantress 500. El alimento fue en base a una dieta estándar de maíz y soya para pollos de engorde, ofrecida ad libitum según la fase de producción: inicio (1-21 días), crecimiento (22-42 días). Las aves fueron vacunadas en la planta de incubación al primer día de edad contra la enfermedad de Marek, bronquitis infecciosa y enfermedad de Newcastle, y en el galpón experimental a los 10 días de edad contra la enfermedad de Newcastle.

Las aves fueron distribuidas en forma aleatoria en cuatro tratamientos con cinco repeticiones de 20 aves cada uno. Los tratamientos fueron: T1: Aves control negativo sin APC en el alimento; T2 y T3: Aves que recibieron enramicina al $8 \%$ en el alimento $(10 \mathrm{y}$ 5 ppm, respectivamente); T4: Aves que recibieron tilosina fosfato al $25 \%$ en el alimento (55 ppm). Ambos antibióticos proceden del laboratorio comercial Ilender Perú (Enroque ${ }^{\circledR}$ y Performance $T 25 \AA$, respectivamente). Las dosis empleadas fueron planteadas de acuerdo con los niveles máximos de uso permitidos por el Servicio Nacional de Sanidad Agraria (Perú) con el fin de garantizar la máxima eficacia en la promoción del crecimiento y rendimiento productivo.
Las aves fueron criadas a galpón abierto, en un mismo ambiente, sobre piso de cemento con cama de viruta de madera, en una densidad de $24 \mathrm{~kg} / \mathrm{m}^{2}$. Las condiciones ambientales durante el experimento se manejaron según la edad de las aves, utilizando cortinas externas para controlar la temperatura ambiental, además de un «cielo raso» como microclima, para facilitar un ambiente de confort a las aves.

Todas las aves fueron pesadas al primer día de edad y en forma semanal. Se utilizó una balanza electrónica JBC modelo JCS$\mathrm{B}$ con capacidad de $30 \mathrm{~kg}$ y precisión de $1 \mathrm{~g}$. Se calculó la ganancia de peso. El consumo del alimento fue registrado semanalmente, el índice de conversión alimenticia (ICA) fue calculado semanalmente según la fórmula: alimento consumido / peso vivo, y el índice de eficiencia productivo europeo (IEPE) se determinó al término del estudio, según la fórmula: (Viabilidad $x$ Ganancia diaria de peso $x$ 100) / ICA. Se registró la mortalidad y se realizó la necropsia de las aves para determinar la causa de muerte.

Los parámetros productivos fueron evaluados mediante análisis de varianza de una vía, con análisis de Bartlet para comprobar la homogeneidad de varianzas entre tratamientos. Comparaciones múltiples entre los parámetros promedio según tratamientos se hicieron mediante la prueba de Bonferroni. En el análisis estadístico se usó el programa R v. 3.3.1.

\section{Resultados}

El peso promedio al primer día de edad fue similar en los cuatro grupos experimentales. El peso final en las aves de T1 (Control) fue significativamente menor que el peso en los grupos tratados (Cuadro $1 ; \mathrm{p}<0.05$ ). Así mismo, las aves de T1 (Control) presenta- 
Cuadro 1. Parámetros productivos en pollos de engorde de la línea Cobb-Vantress 500 a los 42 días de edad, en respuesta a la suplementación con tilosina fosfato y enramicina

\begin{tabular}{cccccccc}
\hline Trat. ${ }^{1}$ & $\begin{array}{c}\text { Peso } \\
\text { corporal } \\
(\mathrm{g})\end{array}$ & $\begin{array}{c}\text { Ganancia de } \\
\text { peso (g/día) }\end{array}$ & $\begin{array}{c}\text { Consu } \\
\text { mo }(\mathrm{g})\end{array}$ & $\mathrm{ICA}^{2}$ & $\begin{array}{c}\text { Mortalidad } \\
(\%)\end{array}$ & $\begin{array}{c}\text { Viabilidad } \\
(\%)\end{array}$ & IEPE $^{3}$ \\
\hline $\mathrm{T} 1$ & $2700^{\mathrm{a}}$ & $64.28^{\mathrm{a}}$ & 4592 & $1.70^{\mathrm{a}}$ & 4 & 96 & $362.86^{\mathrm{a}}$ \\
$\mathrm{T} 2$ & $2830^{\mathrm{b}}$ & $67.35^{\mathrm{b}}$ & 4476 & $1.58^{\mathrm{b}}$ & 4 & 96 & $408.65^{\mathrm{b}}$ \\
$\mathrm{T} 3$ & $2814^{\mathrm{b}}$ & $67.00^{\mathrm{a}}$ & 4558 & $1.62^{\mathrm{b}}$ & 2 & 98 & $405.26^{\mathrm{b}}$ \\
$\mathrm{T} 4$ & $2862^{\mathrm{b}}$ & $68.17^{\mathrm{b}}$ & 4580 & $1.60^{\mathrm{b}}$ & 0 & 100 & $426.46^{\mathrm{b}}$ \\
\hline
\end{tabular}

${ }^{a, b}$ Superíndices diferentes dentro de columnas indican diferencia estadística $(p<0.05)$

${ }^{1}$ T1: sin APC; T2 y T3: enramicina (10 y 5 ppm, respectivamente); T4: tilosina fosfato (55 ppm)

2 Índice de conversión alimenticia

3 Índice de eficiencia productivo europeo

ron el ICA y el IEPE más deficiente $(1.70 \mathrm{y}$ 382.86 , respectivamente), siendo estadísticamente diferente al resto $(\mathrm{p}<0.05)$. Por otro lado, no hubo diferencias significativas entre tratamientos por consumo de alimento, ganancia de peso y porcentaje de mortalidad (Cuadro 1). Se presentó una mortalidad mayor en T1 y T2 asociada principalmente a descarte por cojera y muerte súbita por el rápido crecimiento de las aves. Se encontró diferencia significativa en ganancia de peso diaria (g/día) de T2 y T4 con los demás tratamientos $(\mathrm{p}<0.05)$.

\section{Discusión}

Las aves de T4 obtuvieron un mayor, aunque no estadísticamente significativo peso corporal $(2.862 \mathrm{~g})$ que los grupos T2 y T3. Por otro lado, el peso corporal de T1 a los 42 días fue menor que el peso logrado en los grupos tratados $(p<0.05)$. Estos resultados son mejores que los reportados por Guaranga (2012) quién suplementó enramicina al 8\% a dosis de 75, 100 y $125 \mathrm{~g} / \mathrm{t}$ en pollos de engor- de criados hasta los 49 días de edad, sin encontrar una ventaja significativa con el grupo control.

Las diferencias significativas en ganancia de peso diaria de T2 (10 ppm de enramicina al $8 \%$ ) y T4 (55 ppm tilosina 25\%) en comparación con los tratamiento control y T3 (5 ppm enramicina al 8\%) fueron diferentes a los reportados por Wang et al. (2016), quienes encontraron diferencia significativa al administrar 5 ppm de enramicina en la dieta en comparación con el control; mientras que Kamran et al. (2013) en un experimento similar encontraron diferencias significativas en ganancia de peso al administrar $0.12 \mathrm{~kg} / \mathrm{t}$ de enramicina como promotores de crecimiento comparados contra el grupo control.

El consumo de alimento fue similar en el grupo control en comparación con los grupos tratados; resultado similar al experimento de Rosas (2014); no obstante, Guaranga (2012) obtuvo un menor consumo en los animales suplementados con enramicina al $8 \%$ en dosis de $125 \mathrm{~g} / \mathrm{t}$ ( $80 \mathrm{~g}$ menos de consumo de alimento que el grupo control). 
E1 ICA fue positivamente afectado por el empleo de los promotores de crecimiento; mientras que Rosas (2014) no llegó a encontrar un efecto benéfico con la suplementación tilosina fosfato. En forma similar, el valor de IEPE fue mejor en las aves de los grupos tratados en comparación con el grupo control $(\mathrm{p}<0.05)$, resultado que concuerda con el trabajo de Guaranga (2012) suplementando enramicina en dosis de $100 \mathrm{~g} / \mathrm{t}$ (268.14) en comparación con aves suplementadas con este antibiótico en dosis de $75 \mathrm{~g} / \mathrm{t}$. Por otro lado, otros autores no obtuvieron mejoras en el IEPE al suplementar con Zinc bacitracina en comparación con un probiótico (Osorio et al., 2010) o con fosfato de tilosina a dosis de 40 ppm en comparación con el grupo control (Rosas, 2014).

\section{Conclusiones}

- Los tres grupos suplementados con promotores antibióticos tuvieron mejor peso corporal, índice de conversión alimenticia (ICA) e índice de eficiencia productivo europeo (IEPE) que las aves del grupo control $(\mathrm{p}<0.05)$.

- No hubo diferencias significativas en peso corporal, consumo de alimento, ICA e IEPE entre los tratamientos con $55 \mathrm{ppm}$ de tilosina fosfato y 5 y $10 \mathrm{ppm}$ de enramicina suplementados en el alimento de pollos de engorde.

- Se encontró diferencia significativa en ganancia de peso diaria entre los grupos suplementados con $10 \mathrm{ppm}$ enramicina y $55 \mathrm{ppm}$ de tilosina fosfato en comparación con el grupo control y el tratamiento de 5 ppm de enramicina.

\section{Literatura Citada}

1. Acar J, Rostel B. 2003. Antimicrobial resistance, an overview. In: OIE standards on antimicrobials resistance. Paris, France: OIE. p 45-68.
2. Casewell M, Friis C, Marco E, McMullin P, Phillips I. 2003. The European ban on growth-promoting antibiotics and emerging consequences for human and animal health. J Antimicrob Chemoth 52: 159-161. doi: $10.1093 / \mathrm{jac} / \mathrm{dkg} 313$

3. Cepero R. 2006. Retirada de los antibióticos promotores de crecimiento en la Unión Europea: causas y consecuencias. En: XII Congreso Bienal de la Asociación Mexicana de Especialistas en Nutrición Avícola (AMENA). Puerto Vallarta, Jalisco, México.

4. Cerdá R. 2011. Control de micoplasmas mediante el uso de antibióticos. ¿por qué fallan tan frecuentemente? En: XXII Congreso Latinoamericano de Avicultura. Buenos Aires Argentina

5. European Commission. 2005. Ban on antibiotics as growth promoters in animal feed enters into effect. [Internet]. Available in: http://europa.eu/rapid/pressrelease_IP-05-1687_en.htm

6. Dibner JJ, Richards JD. 2005. Antibiotics growth promoters in agriculture: history and mode of action. Poultry Sci 84: 634-643. doi: 10.1093/ps/ 84.4 .634

7. [FAMIC] Food and Agricultural Materials Inspection Center. 2014. Antibiotics. [Internet]. Disponible en: http://www.famic.go.jp/ffis/oie/obj/ a7_er.pdf

8. Ferket PR. 2007. Controlling gut health without the use of antibiotics. College of Agriculture and Life Sciences, North Carolina State University, USA. [Internet]. Available in: http://citeseerx.ist.psu.edu/ viewdoc/summary?doi=10.1.1.318.7689

9. Guaranga $W$. 2012. Utilización de diferentes niveles de enramicina en dietas para pollos parrilleros. Tesis de Ing. Zootecnista. Riobamba, Ecuador: Escuela Superior Politécnica de Chimborazo. $88 \mathrm{p}$.

10. Kamran Z, Mirzaa MA, Ahmad $S$, Samad HA, Sohail MU, Saadullah M. 2013. Performance of broiler chickens 
fed mannan oligosaccharides as alternatives to antibiotics from one to twentytwo days of age. J Anim Plant Sci 23: 1482-1485.

11. Kim H, Borewicz K, White BA, Singer $R$, Sreevatsan $S, T u Z J$, Isaacson $R E$. 2012. Microbial shifts in the swine distal gut in response to the treatment with antimicrobial growth promoter, tylosin. $P$ Natl Acad Sci USA 109: 15485-15490. doi: 10.1073/pnas.1205147109

12. Lewicki J. 2006. Tylosin. A review of pharmacokinetics, residues in food animals and analytical methods. Tylosin Residue Review FAO. 39 p.

13. Lin J. 2014. Antibiotic growth promoters enhance animal production by targeting intestinal bile salt hydrolase and its producers. Front Microbiol 5: 33. doi: 10.3389/fmicb.2014.00033

14. [OMS] Organización Mundial de la Salud. 2007. Amenazas para la seguridad sanitaria. Ginebra: OMS. Serie de Informes Técnicos. $23 \mathrm{p}$.

15. Osorio C, Icochea E, Reyna P, Guzmán J, Carzola F, Carcelén F. 2010. Comparación del rendimiento productivo de pollos de carne suplementados con un prebiótico versus un antibiótico. Rev Inv Vet Perú 21: 219-222. doi: 10.15381/rivep.v21i2.140
16. Rosas J. 2014. Comparación del rendimiento productiva de pollos de engorde suplementados con tylosina fosfato como promotor de crecimiento en dosis mínima y máxima. Tesis de Médico Veterinario. Lima, Perú: Univ. Nacional Mayor de San Marcos. $50 \mathrm{p}$.

17. Shiva C. 2007. Estudio de la actividad microbiana de extractos naturales y ácidos orgánicos. Posible alternativa a los antibióticos promotores de crecimiento. Tesis doctoral. Barcelona, España: Univ. Autónoma de Barcelona. 173 p.

18. Sumano HS, Gutiérrez L. 2010. Farmacología clínica en aves comerciales. $4^{\text {a }}$ ed. México: McGraw-Hill Interamericana. $703 \mathrm{p}$.

19. Vargas F. 2010. El uso inteligente de promotores del crecimiento puede aumentar las utilidades. [Internet]. Disponible en: http://www.thepoultrysite.com/ intestinalhealth/issue24/latino-amricaedicin-6/211/el-uso-inteligente-de-lospromotores-del-crecimiento-puede-aumentar-las-utilidades/

20. Wang HL, Shi M, Xu X, Pan L, Zhao $P F, M a X$, Tian $Q$, et al. 2016. Effects of flavomycin, Bacillus licheniformis and enramycin on performance, nutrient digestibility, gut morphology and the intestinal microflora of broilers. J Poult Sci 53: 128-135. doi: 10.2141/jpsa.0150 077 